\title{
Mnémosyne à l'époque de la digitalisation globale
}

(Tavola des chocs modernes)

\section{Aurora Fernández-Polanco}

\section{CpenEdition}

\section{Journals}

Édition électronique

URL : http://journals.openedition.org/imagesrevues/2888

DOI : 10.4000/imagesrevues.2888

ISSN : 1778-3801

Éditeur :

Centre d'Histoire et Théorie des Arts, Groupe d'Anthropologie Historique de l'Occident Médiéval, Laboratoire d'Anthropologie Sociale, UMR 8210 Anthropologie et Histoire des Mondes Antiques

\section{Référence électronique}

Aurora Fernández-Polanco, « Mnémosyne à l'époque de la digitalisation globale », Images Re-vues [En ligne], Hors-série 4 | 2013, mis en ligne le 30 janvier 2013, consulté le 30 janvier 2021. URL : http:// journals.openedition.org/imagesrevues/2888 ; DOI : https://doi.org/10.4000/imagesrevues.2888

Ce document a été généré automatiquement le 30 janvier 2021.

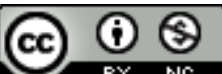

Images Re-vues est mise à disposition selon les termes de la Licence Creative Commons Attribution Pas d'Utilisation Commerciale 4.0 International. 


\section{Mnémosyne à l'époque de la digitalisation globale}

(Tavola des chocs modernes)

\section{Aurora Fernández-Polanco}

Il est semblable à quelque chose mais simplement semblable.

Et il invente des espaces dont il est la «possession convulsive»

(Roger CAILLOIS) 
1 La bande-annonce du film Socialisme (2010) de Godard montre que nous vivons la modernité sur le mode de l'excès : excès de vitesse, d'images, de bruit et d'urgence $^{1}$. L'expérience du choc qui, au début du XXe siècle, n'était qu'urbaine, est aujourd'hui devenue numérique dans notre environnement Web. Et c'est cette expérience que transmet la bande-

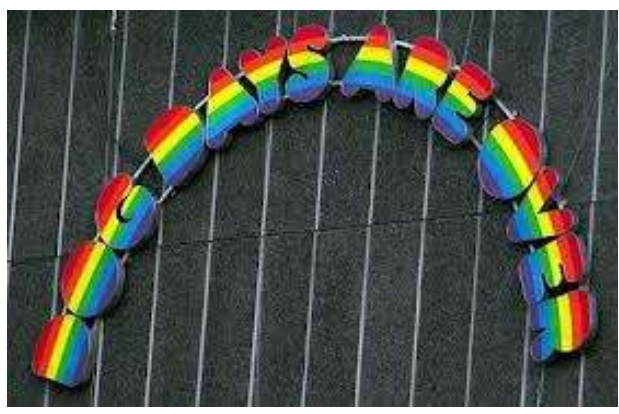
annonce $d u$ film, expérience "épileptique », peut-on lire dans Cahiers du cinéma, " jetée dans la mer, dans l'océan du Net ». Le film monté en accéléré et entièrement fait de petits fragments, de petits chocs produits par la vitesse des images, "fait rimer hypervisibilité et invisibilité ${ }^{2}$. Mais peut-être Godard nous avait-il déjà fait entrevoir l'avenir avec « la valise-inventaire » du monde emplie d'images, que les jeunes carabiniers, dans son film de 1967, lançaient au vent. Jouissance, dans une foi naïve, de tout voir ou de tout avoir ? Conscience, chez ces jeunes gens, d'avoir été le jouet d'une farce, d'où le tourbillon, l'intentionnel chaos des images? Où mélancolie pour nous, aujourd'hui, après coup, de n'avoir rien conservé et de constater, comme le dit Guy Debord, que «tout ce qui était directement vécu s'est éloigné dans une représentation ». Tel serait notre nouveau destin phobique, résultant de la perte de la distance, l'état précisément d'où Warburg était parti dans son travail sur le rituel du serpent. Là-bas, l'homme primitif, au milieu de la nature menaçante ; ici, nous, dans la culture de l'excès, de la disponibilité immédiate, au milieu d'un chaos agressif. Nous connaissons la réticence de Warburg vis-à-vis de la technique moderne : «Franklin et les frères Wright, qui ont inventé le ballon dirigeable, sont les destructeurs fatidiques de la notion de distance ». Fin de la zone de contemplation et de l'espace de pensée (Denkraum), cet «espace que la communication électrique instantanée anéantit $»^{3}$. Warburg, à l'instar de Simmel, Kracauer et Benjamin ${ }^{4}$, a donc pensé les relations entre technique et modernité à partir du concept de distance.

2 Philippe-Alain Michaud, en réponse à Gombrich, soutient que Warburg « reprend les clichés du positivisme pour les renverser... » car les peurs primitives "ne s'opposent pas à la connaissance, mais au désenchantement du monde que l'historien de l'art diagnostique comme le signe définitif de l'instrumentalisation de la nature, dans la disparition des relations spatiales et la domestication de l'énergie ${ }^{5}$. Mais les peurs primitives reviennent et s'installent dans ce monde technique, monde-web des appels infinis, monde du «direct» (comme lors de l'écroulement des tours jumelles le 11 septembre), de la «communication électrique instantanée ${ }^{6}$. Pour penser cette expérience de l'excès et $\mathrm{du}$ " trop direct », il faudrait retracer sa préhistoire et pour cela reprendre la lecture faite par Susan Buck-Morss d'une citation de Benjamin: «L'environnement altéré par la technologie expose le sensorium humain à des chocs physiques qui trouvent leur correspondance dans le choc psychique $»^{7}$.

3 En 1939, en pleine guerre, l'année même de la publication de "Sur quelques thèmes baudelairiens ", le philosophe espagnol Ortega y Gasset donnait une conférence à Buenos Aires intitulée: Ensimismamiento y alteración ${ }^{8}$ (concentration et trouble), à un moment où personne ne pouvait se concentrer (ensimismarse: littéralement "s'enfermer en soi ») : «Tout le monde est troublé - dit Ortega -, le trouble nous aveugle, nous oblige à agir mécaniquement dans un état de somnambulisme frénétique $»^{9}$. Le philosophe 
nous envoie au zoo et nous demande de rester debout devant la cage des singes. "Tout à coup, dit-il, nous comprendrons quelque chose comme en un éclair. Quel destin d'être inquiet, d'être sans repos que le nôtre, quel mélange de peur et volonté de tout avoir !» L'homme troublé est perdu au milieu d'un monde qu'il ne comprend pas; pris dans l'immédiateté des choses, il n'a pas la possibilité de penser, il se trouve dans l'état phobique dont parle Warburg. Mais, disait Ortega, il peut «suspendre son commerce direct avec les choses», se libérer de leur emprise et tourner le dos au monde, et alors se montrer capable de se concentrer sur soi-même (ensimismarse), de revenir à soi-même pour commencer à penser. Tandis que l'animal vit toujours sous la domination des stimuli, l'homme peut faire des allers-retours, se perdre dans le monde et retourner en soi pour planifier la façon d'humaniser le monde. Mais, pour prendre les mots de la clinique, quand ce mouvement dialectique - trouble/concentration- s'interrompt, il se retrouve ou face à la manie ou face à la dépression. L'omnipotence maniaque ou bien l'impotence de la dépression ${ }^{10}$.

Dix ans avant l'analyse d'Ortega, dans une note écrite peu avant sa mort - une note très souvent citée -, Aby Warburg définit la nouveauté de sa position en ces mêmes termes cliniques antagonistes: "Souvent, il me vient à l'esprit que, en tant que psychohistorien, je cherche à établir la schizophrénie de la civilisation occidentale à partir de ses images par un réflexe autobiographique : la nymphe extatique (maniaque) d'un côté, et le dieu fluvial mélancolique (dépressif) de l'autre $»^{11}$.

En m'appuyant sur cette polarisation, je souhaiterais examiner deux œuvres vidéo héritières, en quelque sorte, de l'identification du monde de la création (Künstler) léguée par la tradition romantique. Je m'arrêterais sur la figure du saltimbanque entre clown et mime -, figure solidaire de la crise de la perception située par Benjamin au temps de Baudelaire et devenue aujourd'hui celle des désordres de l'attention et du désengagement. Il ne s'agit pas retracer l'histoire de cette figure, mais de prendre deux exemples artistiques contemporains qui la réactualisent et incarnent la tension indiquée par Warburg dans son Tagebuch.

La première vidéo est une installation de Bruce Nauman, Clown Torture, datant de 1987 : ce serait notre nymphe maniaque. La seconde, Dogs day Are Over ${ }^{12}$, installation d'Ugo Rondinone (1996) serait l'équivalent du dieu fluvial mélancolique. Dans la vidéo de Nauman, on voit un clown qui ressasse une quinzaine de fois la même histoire en boucle, sans début ni fin. Cacophonie d'images et de sons impossible à supporter. Epuisé, le clown finit par tomber. Il est possible que le monde infernal de la répétition renvoie à la modernité, époque du « toujours, toujours, toujours » et que le rythme des mots soit l'imitation du bruit de la machine, comme l'avait indiqué Michelet ${ }^{13}$. En tout cas, le clown de Nauman, même s'il est une victime, à encore la capacité d'agir et de nous faire rire. Ceux de Rondinone, en revanche, sont immobilisés dans une posture proche de la mort évoquant l'état de dépression décrit par Pierre Fédida : «il convient aussi de penser cet état [...] comme la mise en conservation du vivant sous une forme inanimée», «une sorte de protection paradoxale contre son propre anéantissement $»^{14 .}$ Détachés du monde, les clowns ont perdu le pouvoir de regarder et la faculté de faire rire.

7 Eloignons-nous de la représentation topique de l'artiste en saltimbanque, telle que Jean Starobinski ${ }^{15} \mathrm{a}$ pu la fixer, pour rejoindre la figure proposée par Warburg de l'artiste en funambule, cette petite figure que G. Didi-Huberman a caractérisée comme celle de la balançoire éternelle. Dans la situation d'excès dont nous parlions en commençant, nous 
pouvons ressentir une certaine empathie avec ces clowns. Je me permets donc de manipuler un peu Warburg et Didi-Huberman pour repenser cette figure dans le monde Web, et du côté de l'artiste et du côté du spectateur, en un temps où le format www mélange la production et la réception. Ne sommes-nous pas nous-mêmes des funambules dans la mesure où notre désir de tout voir, de tout entendre, de tout collectionner s'inverse en un mouvement contraire ? D'un côté, la manie, de l'autre, la mélancolie devenue dépression, disengagement ${ }^{16 .}$ Les désordres de la dépression n'ont rien à voir avec l'impuissance mélancolique, problème tenant à la "performance de l'œuvre ", mais avec un problème tenant à la " performance du soi »" Dans une culture d'après les années 70 , dont les normes sociales sont fondées sur la " création du soi», on parle en général d'inhibition, de fatigue et de diminution des activités cognitives orientées vers un but. Dans le texte The Aesthetics of Disengagement, C. Ross examine les œuvres d'artistes qui travaillent cet état de choses.

8 Après cette courte introduction, j'aimerais rester avec les clowns et les fixer à la tavolaécran - c'est par le mot tavola qu'on traduit en espagnol le terme panel employé par Gombrich, soit en français "écran d'exposition». Une tavola encore in fieri ${ }^{18}$ qui recueille l'image des symptômes nerveux modernes, de la «modernité de la neurasthénie ", de la " maladie de la vie moderne qui résulte de la trépidation des temps nouveaux et qui représente la dimension nerveuse de la fatigue industrielle $»^{19}$. Sorte d'archive historique des intensités " modernes ", pour reprendre l'expression de Warburg, mais cette fois-ci perceptives, au sens où Walter Benjamin, qui avait fait du choc « l'essence même de l'expérience moderne ${ }^{20}$, avait compris la modernité comme crise de la perception. Archive des chocs subis sous les coups d'une vie moderne altérée par la technique.

9 Sur la «tavola des chocs subis", nous ne sommes pas confrontés à l'expression organique du pathos, mais au moment d'implosion où, comme l'a évoqué Giorgio Agamben, la bourgeoisie fin de siècle a "perdu ses gestes ${ }^{21}$ et où le corps se trouve réifié comme instrument de travail et comme marchandise. Ce n'est pas un hasard si Hofmannsthal écrit en 1893: "Ce qui est moderne ce sont les vieux meubles et les jeunes nervosités " ${ }^{22 .}$ L'article " Notes sur le geste », de Giorgio Agamben, paru pour la première fois dans la revue cinématographique Trafic, peut être considéré comme un texte-montage. Dans cet article, Agamben relie d'une certaine façon Warburg au cinéma dans la mesure où l'un comme l'autre « ramènent les images dans la patrie du geste ${ }^{23 .}$. Agamben parle de la catastrophe généralisée du geste, du syndrome de Gilles de la Tourrette (quand les muscles semblent danser), de la dysbasie, de l'apraxie, des lapsus d'action - et il reconnaît qu'au moment même où l'homme perd ses gestes, il commence à s'interroger sur eux, à les étudier et à être obsédé par eux.

10 Je ne présente ici qu'un rapide exposé de mon objet d'étude ${ }^{24}$. Je sais qu'une collection iconographique ne suffit pas pour travailler dans l'esprit de l'Atlas Mnémosyne. Prises au sens warburgien, les images évoquées ne pourront être " déchiffrées que comme un symptôme, c'est-à-dire comme le symbole mémoriel d'émotions passées ${ }^{25}$. Pour l'instant, passant outre les problèmes soulevés par le choix des images, contentonsnous de garder la tavola comme un espace analytique, comme un " espace de pensée ». De fait, cette collection de corps "saccadés $»^{26} s^{\prime}$ allie très bien avec la méthode du montage comme forme de pensée. Donc, sur cette tavola-écran, je commence par accrocher avec des épingles la vidéo-installation d'Aernout Mik, Middlemen (2001). 
11 J'ai d'abord « vu » dans Middlemen les tics du Charlot des Temps Modernes et, à partir de là, j'ai ressenti le besoin, pour écrire, de «monter » les images de chaplin avec certaines images d'Aernout Mik. Chez ce dernier, il n'y a pas d'usine, pas de machines, mais des images de brokers de New York, ainsi que l'explique Sabine Maria Smidt, une des commissaires de l'exposition du Jeu de Paume (2011): «Les interprétations contemporaines ont souvent vu dans cette œuvre une annonce prophétique de la récente crise financière. Une Bourse dont le sol est jonché de papiers, courtiers (les middlemen) au bord de la crise de nerfs, abattus ou secoués de tics - l'impression de débâcle ou de récente catastrophe est dominante -. La scène est entièrement construite par l'artiste, du décor aux gestes et attitudes des acteurs ${ }^{27}$. Pour Sarah Ilher-Meyer :

«Un travelling lent et continuel nous fait cheminer dans une salle des marchés parmi des brokers et des courtiers, les uns affairés, les autres abattus, assis sur des marches jonchées de papiers, le regard perdu et (je souligne) le corps parcouru de soubresauts. Un crash boursier vient sans doute de se produire, mais nulle information sur son contexte ni aucune logique narrative ne vient en éclairer les raisons. Peut-être précisément parce que, tout aussi explicable que soit une catastrophe économique, son avènement n'a rien d'inéluctable, ses acteurs ayant eu la possibilité de prendre une trajectoire différente ».

12 Il me paraît fort intéressant que ces hommes soient en même temps abattus et secoués de tics, le corps « parcouru de soubresauts » et que certains d'entre eux présentent une ressemblance troublante : en réalité, « l'un d'eux est un automate, qui double les gestes et expressions de l'autre $»^{28}$.

Pourquoi ai-je «vu » le Charlot des Temps Modernes, prototype de l'homme «assemblé mécaniquement (einmontiert) dans la structure du film », en me rappelant les mots de Benjamin? Telle est bien la nouveauté de son geste (gestus) : "il décompose les mouvements expressifs de l'homme en une suite d'innervations infimes. Chacun de ses mouvements singuliers se compose d'une suite en saccade de mouvements fractionnés. [...] c'est toujours la même succession saccadée de mouvements minuscules qui érige la loi de la succession des images filmiques au rang de loi de la motricité de l'homme ${ }^{29}$. Désormais, ce n'est plus la figure « organique » du singe qui nous accompagne, comme avec Ortega y Gasset. Charlot est réifié comme une marchandise, comme s'il avait perdu ses gestes organiques et qu'il cherchait à se protéger du choc mécanique par un mimétisme sympathique, de type magique. «La mimésis n'est nulle part plus apparente comme réflexe défensif qu'à l'usine, où (Benjamin cite Marx) « les travailleurs apprennent à adapter leurs mouvements au mouvement continu et uniforme de l'automate »[...] uniformité de vêtement et de conduite, mais uniformité aussi de mimique [...] le sourire joue un rôle d'amortisseur mimique [...]. L'effet sur le système cénesthésique est brutalisant. Les facultés mimétiques, plutôt que d'incorporer le monde extérieur comme forme d'encapacitation [empowerment] [...] sont utilisées comme barrage contre lui. Le sourire qui apparait automatiquement sur le visage du passant évite le contact $»^{30}$.

On trouve un exemple plus proche du capitalisme post-fordiste et informationnel voilà une nouvelle image à accrocher sur la tavola -, avec une installation multi-écrans qui entre en dialectique avec Les Temps modernes de Chaplin et avec Middlemen d'Aernout Mik et qui se laisse lire comme symptôme, "symbole mémorial des émotions passées ». Je parle d'Electric Earth, de Doug Aitken, qui nous montre le danseur hip-hop Ali Johnson dans un paysage désert de banlieue industrielle, véritable mime doté d'une surcapacité kinesthésique : «Souvent, je danse si vite que je deviens ce qu'il y a autour 
de moi. Pour moi, c'est comme de la nourriture. J'aime absorber cette énergie, cette information. C'est comme si je les mangeais. C'est le seul talent que j'ai $»^{31}$.

Pour Electric Earth, « Doug Aitken a conçu un trajet en trois salles, dont celle du centre est divisée en deux. Chaque salle correspond à un moment du récit déployé dans l'espace et le temps, bien que les séquences en boucle donnent le sentiment d'une clôture événementielle fortement marquée. Mais le son de la salle suivante appelle le visiteur à continuer son parcours. Celui-ci est rythmé par les parois de chaque salle qui font office de coupes franches équivalentes à celles du montage. L'architecture a une fonction narrative, d'autant plus évidente, que la salle centrale est divisée en deux, offrant ainsi un effet d'enchaînement en surimpression entre deux projections : l'œil du visiteur capté par une "séquence", voit simultanément au fond de la salle une autre "séquence" séparée mais néanmoins reliée par la transparence brouillée d'une cloison. La troisième salle offre enfin une ultime séquence dont l'événement représenté impose au spectateur visiteur un sentiment de fin : un personnage s'éloigne dans le lointain d'un tunnel $»^{32}$.

Le spectateur est pris dans un environnement de "tactilité optique »; l'être mimétique attiré par l'espace, se perd dans l'espace. Selon les propos de Taussig relayant les analyses de Caillois, la faculté mimétique est alors « le "degré zéro" de la ressemblance, une plasticité ineffable face aux formes du monde et de la vie ». «Je prends une position extrême pour comprendre ce qui est précisément en jeu avec la faculté mimétique, ce "degré zéro" de similitude, cette plasticité ineffable face aux formes du monde et aux formes de la vie» ${ }^{33}$, dit Taussig qui cite aussi Benjamin quand il parle d'optical tactility et indique comment la sensibilité nous porte hors de nous : «il n'y a pas de proposition plus adéquate pour comprendre le lien viscéral connectant le percepteur et le perçu [...] dans l'opération de la mimesis $»^{34}$.

Mais revenons au mimétisme comme forme de survie et comme possibilité d'agir pour contrôler son environnement, avec un autre exemple, différent des précédents. Pour Warburg, il n'est nul besoin de distinguer temps et espace, c'est pourquoi nous pouvons travailler à sa manière, comme des sismographes «sur la ligne de partage entre les cultures ${ }^{35}$. Continuons donc sur cette ligne de partage, en emboîtant le pas de ses intérêts anthropologiques. Nous rencontrons l'ethno-cinéaste Jean Rouch, «l'homme qui, à la fin des années 1940, avait introduit, avec une insouciante témérité, le film 16 $\mathrm{mm}$ en anthropologie, cette "discipline faite de mots" selon l'expression de Margaret Mead $»^{36}$. Sauf que, dans ce cas-là, on ne peut parler de sismographe puisque la caméra de Rouch « s'immisce au cœur de l'action, la modifie, la provoque, crée la réalité qu'elle décrit. [...] l'enquête l'affecte et il affecte l'enquête : en filmant, il s'absorbe dans une ciné-transe qui le place au centre du rituel $\aleph^{37}$. Avec Rouch, nous sommes au-delà du mimétisme de Chaplin, des brokers de Middlemen ou du danseur d'Electric Earth car nous revenons aux spasmes brusques et involontaires des corps qui se sont soumis à « l'incorporation ». Je parle du rituel de possession, du mimétisme et de la "prise de chair ", montrés dans le film Les Maîtres fous de 1954. Il s'agit d'un phénomène de possession dans un contexte rituel et urbain (je souligne) ; une fois encore, donc, c'est la relation entre capitalisme et technique qui est en jeu.

Dès ses débuts, Jean Rouch a prêté une attention toute particulière aux images. Mais pas dans leur dimension esthétique. Proche de Warburg en ce sens - ou, du moins, de ses sentiments -, Rouch aimait tout ce que les images peuvent avoir de magique. Je cite ses propres mots, à l'âge de 17 ans : 
19 «Au coin du Boulevard Montparnasse et du Boulevard Raspail au printemps 1934 [...]. Dans la vitrine d'une librairie que le soleil de fin d'après-midi illuminait d'un éclairage rasant, étaient exposées deux grandes pages de la Revue Minotaure. L'une extraite du numéro spécial de cette revue consacrée à la mission Dakar Djibouti, était la photo inoubliable des masques Kanaga montés sur la terrasse du chasseur Monze pour son dama, l'autre était le frontispice du numéro 5 de mai 1934 de la peinture métaphysique de Giorgio de Chirico, le Duo ou les Mannequins de la Tour rose. Tout d'un coup c'était la rencontre du merveilleux, aussi bien dans la photographie de Marcel Griaule des Dogon de la falaise de Bandiagara que dans ces deux personnages emmaillotés d'inquiétude et eux aussi montés sur une terrasse au soleil couchant...». ${ }^{38}$

Nadine Wanono Sonchamp évoque aussi Warburg à propos de "cette fusion entre ses intensités lumineuses [...], cette illumination du soleil couchant qui regroupe tout à la fois la lumière de cette journée de mai 1934, le tableau de G. de Chirico et la photo de Marcel Griaule $\aleph^{39}$. On pourrait aussi dire qu'il s'agit de l'imagination en action, au sens baudelairien.

\section{Imiter la technique (des colonisateurs) pour la domestiquer}

21 Dans Les Maîtres fous ${ }^{40}$, Rouch filme en un seul jour les pratiques de possession des Haouka, « les maîtres de la folie », une secte constituée dans les faubourgs d'Accra et de Kumasi au cours des années $20^{41}$. Le film commence par ces mots :

«Venus de la brousse aux villes de l'Afrique Noire, de jeunes hommes se heurtent à la civilisation mécanique. Ainsi naissent des conflits et de nouvelles religions. Ainsi s'est formée, vers 1927, la secte des Haouka. Ce film montre un épisode de la vie des Haouka de la ville d'Accra. Il a été tourné à la demande de prêtres, fiers de leur art Mountyeba et Moukayla. Aucune scène n'en est interdite ou secrète mais ouverte à ceux qui veulent bien jouer le jeu. Et ce jeu violent n'est que le reflet de notre civilisation ».

Après la lecture de ce texte, une locomotive traverse le plan en diagonale et la voix de Jean Rouch en off s'élève. Il commence par expliquer comment des hommes, arrivés à Accra (Niger, actuel Ghana) depuis toute l'Afrique Occidentale, occupent des emplois de dockers, contrebandiers, porteurs, manœuvres, bergers, marchands de troupeaux, etc. Il nous parle d'une Babylone noire, ville où le bruit et le trafic ne s'arrêtent jamais. Il explique comment, tous les samedis et tous les dimanches, des cortèges de toutes sortes parcourent la ville : "Alors devant ces bruits, devant ces fanfares, les hommes venus des calmes savanes du Nord doivent se réfugier dans les faubourgs de la ville. Et là, tous les dimanches soirs, ils se livrent à des cérémonies que l'on connaît encore très mal : ils appellent les dieux nouveaux, les dieux de la ville, les dieux de la technique, les dieux de la force, les Haouka $»^{42}$.

Le film nous montre que le rituel de possession est une forme de résistance à la colonisation (les jeunes gens noirs imitent l'homme blanc pour le domestiquer, pour le contrôler), mais surtout une tentative pour construire une mémoire de groupe, une mémoire incarnée et capable d'habiter le présent ${ }^{43}$. Mais, il faut le noter, ce qui rend alors possible l'imitation est la possession corporelle. Les Hauka ont demandé à Rouch de filmer eux-mêmes le rituel et le réalisateur a observé qu'ils utilisaient la caméra comme s'il s'agissait d'un outil de bois. Ce sont les dominés qui pratiquent les rituels 
d'inversion et qui ont besoin de la mimesis ; dans le même temps, ils sont européens et non européens.

Les Haouka ne sont plus une " culture primitive ». Ce ne sont pas les Bafiotes dont parle Warburg, qui prend l'exemple de l'assimilation d'une locomotive avec un hippopotame pour illustrer $«$ la substitution objective $»^{44}$. Jean Rouch pour sa part affirme : «Ce n'était plus le génie du tonnerre ou l'arc-en-ciel qui venaient parler avec les hommes, mais le général ou le conducteur de locomotive $»^{45}$.

Dans Les Maîtres fous, il ne s'agit pas de maîtriser techniquement la nature ni de faire " venir l'éclair » comme chez les Hopi, « en se l'appropriant par mimétisme »"46, mais de faire venir « l'oncle Sam », autrement dit les colonisateurs, la technique moderne, en les mimant, en se laissant posséder par eux. Ce qui compte sont moins la bouche écumante et les yeux révulsés des possédés que le fait de se trouver devant l'appropriation technique de la technique au moyen de la technique (le cinéma).

Mathew Rampley ${ }^{47}$ a discerné chez Warburg et Benjamin l'existence d'un même intérêt face à la distance et à l'impact de la technologie dans le monde moderne. Et pour eux, le mimétisme était aussi une notion très importante. Warburg avait subi l'influence d'approches anthropologiques avant la lettre (de Vico à Herder), qui faisaient du mimétisme la condition humaine première, la preuve de l'absence de distance critique chez les hommes primitifs.

Il est indispensable d'envisager l'anthropologie de la ressemblance dans un sens large, comme le montre G. Didi-Huberman au travers de son intérêt pour les phénomènes de métamorphose mimétique. Warburg lui-même, selon Kenneth Clark, avait le don de l'imitation ; il pouvait littéralement «incorporer» un personnage ${ }^{48}$. Et il tenait aux concepts anthropologiques pour comprendre l'expérience humaine, sous la forme d'une polarisation. On est alors loin du vieux concept occidental de représentation, mais proche des jeux de l'enfant, de la danse rituelle; on se trouve dans un espace où l'humanité a perdu l'aptitude à instaurer la distance entre sujet et objet. Il est important de rappeler que la mimesis constitue un concept fondamental de l'épistémologie benjaminienne ${ }^{49}$. Comme Taussing l'a suggéré, on pourrait lire « cette étrange conjonction coloniale de l'homme avec la caméra dans la moitié du siècle XX ${ }^{\text {ème }}$ ${ }^{50}$ à la lumière de son petit article "Sur le pouvoir de l'imitation ", où il est dit que " l'enfant ne joue pas seulement à être épicier ou instituteur, mais aussi à être moulin ou locomotive $»^{51}$.

Mais je voudrais revenir à un autre passage du texte de Benjamin : "Semblable à la flamme, la part mimétique du langage ne peut apparaitre que sur un certain support. Ce support est l'élément sémiotique. Le sens tissé par les mots ou les phrases constitue ainsi le support nécessaire pour qu'apparaisse, avec la soudaineté de l'éclair, la ressemblance. Car celle-ci est souvent, et surtout dans les cas importants, produite - et perçue - par l'homme comme une illumination instantanée. Elle surgit et s'évanouit aussitôt $\aleph^{52}$. Peut-on parler d'une ressemblance entre les extraits filmiques de Chaplin, d'Aernout Mik ${ }^{53}$, d'Aitken et les " Maîtres fous »? Tout au moins suis-je persuadée (tombée que je suis sous le charme d'une certaine «dimension évocatrice ») que c'est " le sens tissé " par le langage du montage propre à " la machine mimétique de la modernité $~^{54}$ et au mimétisme de certaines « formules du tic » qui se montre dans les images de ma tavola, que c'est lui qui produit un effet perçu « comme une illumination instantanée » : « Elle surgit et s'évanouit aussitôt » ${ }^{55}$. 
29 En l'occurrence, le support ou l'élément sémiotique dans lequel la part mimétique du langage se manifeste, c'est l'image filmique. Il n'y a pas de mots chez Chaplin, pas davantage chez Aernout Mik ou Doug Aitke. En ce qui concerne Les Maîtres fous, on ne comprend pas les mots ; il n'y a que la voix de Rouch qui raconte :

«Moukayla, l'Haouka qui l'aidait, proposa non pas de traduire mais d'interpréter tous ces dialogues, non pas mot a mot, mais phrase à phrase. Cette langue était une «glossolalie», une langue qu'on ne pouvait pas traduire, qu'il fallait interpréter, et qui est très utilisée dans les rituels «pentecôtistes» : les Haouka parlaient ainsi la langue du Saint-Esprit. Voilà l'interprétation de Moukayla, l'homme tranquille: ["tant de mystiques dont la glossolalie, q (ui) apparaît au vulgaire comme l'émission déréglée de sons inarticulés, prodrome de la folie, constitue pour les initiés un langage substantiel et enivrant, où l'épel (sic.) d'initiales isolées, une à une, évoque des réalités spirituelles, simples et éternelles, dont les mots ordinaires ne sont que l'image complexe, kaléidoscopique, brouillée, ternie"] $»^{56 .}$

31 On ne s'étonnera guère que Jean Rouch ait dit en regardant les images : " J'avais très curieusement l'impression de vivre une nouvelle expérience de théâtre de la cruauté ${ }^{57}$. Artaud lui-même avait précédemment affirmé que « le cinéma dégage un peu de cette atmosphère de transe $\|^{58}$ et Rouch, de son côté, que « si on montre à quelqu'un un film de lui-même en transe c'est comme un électrochoc $»^{59}$.

32 Assurément, les spectateurs du rituel, devant une brutalité tranquille et continue, étaient émus, mais d'une émotion calme. Sur l'écran, en revanche, c'est une succession de coups de poing ${ }^{60}$ par les effets du montage. Avec le montage - et l'inconscient optique $^{61}$ - on est soumis à la magie mimétique du film et à des illuminations instantanées, grâce aussi à " l'interaction entre les corps mimétiques et la machine mimétique $»^{62}$. Il y a dans le film un moment très spécial : la caméra suit le rituel et, en même temps, Rouch (le montage) présente la parade réelle de l'armée britannique devant l'Assemblée d'Accra. Mais nous sommes ramenés brutalement au rituel. Bhabha a parlé du besoin de la mimique et pour le colonisateur et pour le colonisé. Le colonisateur a eu besoin de se construire une image de lui-même comme possesseur d'une culture supérieure au travers de toute une mise en scène de circonstance, pleine de pompe. Avec un jaune d'œuf, le colonisé mime ironiquement la mimique paranoïaque des colonisateurs ${ }^{63}$.

33 Ce n'est pas un hasard si toutes les images de ma tavola des chocs modernes sont des extraits filmiques. Double choc, mais aussi double jeu, dans l'énigme du double mimétisme. Il y a d'abord le mouvement mimétique de Charlot avec la machine, puis les doubles automates de Middlemen et $\mathrm{du}$ « phasme-Aitken » qui miment l'environnement et, pour finir, la pantomime des colonisateurs jouée par les maîtres fous. Au cinéma, on ne compte pas seulement avec l'effet du montage et l'inconscient optique ; il s'agit aussi d'une projection. Au début du film, Rouch parle du jeu comme « le reflet de notre civilisation ». À la fin ${ }^{64}$, il reconnaît qu' " en comparant ces visages [souriants] avec les visages horribles de la veille, on ne peut s'empêcher de se demander si ces hommes d'Afrique ne connaissent pas certains remèdes qui leur permettent de ne pas être des anormaux, mais d'être parfaitement intégrés à leur milieu, des remèdes que nous ne connaissons pas encore ${ }^{65}$. Quelques années après, il confessé qu'il n'aimait pas cette fin. On sait que le film a suscité beaucoup de critiques depuis sa première présentation au Musée de l'Homme en 1954, en particulier de la part de Marcel Griaule, mais aussi d'autres jeunes étudiants africains. Plus tard, Ousmane Sembène a accusé Rouch 
d'observer les africains comme des insectes ${ }^{66}$. Plus proche de nous, le cinéaste malien Manthia Diawara a dédié à Jean Rouch le film Rouch in reverse (1995) où il parle d'une anthropologie à l'envers. Quoi qu'il en soit, «toutes ces expressions de mimesis, fort appréciées par Taussig, et de mimique, mises fréquemment en évidence par Homi Bhabha, offrent un miroir - oblique, ironique, allégorique, indirect, opaque - de la hiérarchie postcoloniale ${ }^{67}$ : ainsi en est-il dans Les Maîtres fous. L'emploi du mot «toutes" nous fait de nouveau penser à Homi K. Bhabha qui parle "d'une culture internationale, fondée non pas sur l'exotisme du multiculturalisme ou la diversité des cultures, mais sur l'inscription et l'articulation de l'hybridité de la culture » et qui nous avertit: "nous devrions nous rappeler que c'est cet "inter" - le tranchant de la traduction et de la négociation, l'espace entre-deux - qui porte le poids de la signification de la culture [...]. En explorant ce tiers-espace, nous pouvons éluder la politique de polarité, pour une autre politique, et enfin émerger comme les autres de nous-mêmes $»^{68}$.

Nous voici arrivés à la fin. J'espère avoir montré que, même dans l'univers du Web, l'image à l'ère internationale au sens de Bhabha, l'image partagée (au sens donné au mot partage par Susan Buck-Morss) ${ }^{69}$ constitue, comme nous l'apprenons avec Warburg, " un "phénomène anthropologique total", une cristallisation, une condensation particulièrement significative de ce qu'est une "culture" ${ }^{70}$.

\section{NOTES}

1. Je remercie beaucoup Sabine Forero-Mendoza pour son aide dans la rédaction de la version française de ce texte

2. J. Lepastier, "Avènement de socialisme" in Cahiers du Cinéma, Avril, 2010, p.46

3. A. Warburg, Le rituel du serpent : récit d'un voyage en pays pueblo, Paris, Macula, 2003, p. 133: «La pensée mythique et la pensée symbolique, en luttant pour donner une dimension spirituelle à la relation de l'homme à son environnement, ont fait de l'espace une zone de contemplation ou de pensée, espace que la communication électrique instantanée anéantit $»$.

4. Voir Stéphane Füzesséry et Philippe Simay, Le choc des métropoles : Simmel, Kracauer, Benjamin. Paris, Éds. de l'Éclat, 2008.

5. P.-A. Michaud, Aby Warburg et l'image en mouvement, Paris, Macula, 1998, p.223.

6. « One can only imagine how much Warburg would have hated the computer - and the internet in particular". D. Freedberg, Pathos at Oraibi: What Warburg did not see www.columbia.edu/cu/.../Pathos-at-Oraibi.pdf

7. S. Buck-Morss, Voir le capital. Théorie critique et culture visuelle, Paris, Les Prairies ordinaires, 2010.

8. J. Ortega y Gasset, "Ensimismamiento y alteración" (1939) in Obras completas, T.V, Madrid, Revista de Occidente, 1983.

9. Toutes les citations d'Ortega dans cet article sont traduites par l'auteur. 
10. L. Binswanger, Mélancolie et manie. Études phénoménologiques, Paris, Presses Universitaires de France, 1987.

11. Cité par Gombrich du Tagebuch, le 3 avril 1929. Voir G. Didi-Huberman, L'image survivante, Histoire de l'art et temps des fantômes selon Aby Warburg, Paris, Les Éditions de Minuit, 2002 p. 455.

12. Vidéo-installation de 7 canaux sur 7 moniteurs (couleur, sans son), détecteurs audio. Dimensions variables.

13. «[...] c'est le mot invariable que tonne à notre oreille le roulement automatique dont tremblent les planches », cité par W. Benjamin, in Livre des Passages, Paris, Éd. du Cerf, 1989 (D45).

14. P. Fédida, Des bienfaits de la dépression. Éloge de la psychothérapie, Paris, Odile Jacob, 2003, p.19.

15. Je fais allusion au Portrait de l'artiste en saltimbanque, Genève, Skira, 1970.

16. C. Ross, The Aesthetics of Disengagement. Contemporary art and depression, University of Minnesota Press, 2006 : «L'art explore la dépression non simplement comme une maladie, un déficit, une distorsion ou un comportement inadapté mais également comme une forme potentielle de créativité et d'adaptation (...) La contribution originale de l'art au débat contemporain sur la dépression se situe fondamentalement dans sa préoccupation pour le sujet, lequel est ici mis en scène et interpellé suivant un paradigme de dépressivité et dont les symptômes dépressifs sont examinés comme faisant partie de la constitution même de l'image ». http://www.webbynerd.com/artifice/dossierarchives/135.htm

17. Alain Ehrenberg, La fatigue d'être soi : dépression et société, Paris, Poches Odile Jacob, 1998.

18. En ce sens, je me reconnais dans l'analyse de Sigrid Weigel: «C'est surtout l'atlas d'images qui, parce qu'il présente sa collection de gestes dans les domaines de l'art et d'autres médias visuels sous l'aspect d'une mémoire sociale, a contribué à ce que le nom de Warburg soit aujourd'hui encore connu, et ce au-delà des frontières de l'histoire de l'art ", Sigrid Weigel, « Le Rituel du Serpent d'Aby Warburg. Correspondances entre la lecture de textes culturels et de textes écrits " in Trivium, 10, 2012. http://trivium.revues.org/4125 (page consultée le 02/02/2012). Édition originale in : Paragrana, 3, 1994, pp. 9-27.

19. A. Ehrenberg, op.cit., p. 3.

20. S. Buck-Morss, op. cit., p. 153

21. G. Agamben, « Notes sur le geste », in Moyens sans fins, Paris, Payot \& Rivages, 1995.

22. «Gabriele d'Annunzio » [1893], cité par A. Ehrenberg, op. cit., p. 39.

23. G. Agamben, op. cit.

24. ${ }^{23}$ Dans le project «Imágenes del arte y reescritura de las narrativas en la cultura visual global» (HAR2009-10768) (www.imaginarrar.net), nous mettons au point une méthodologie pour « penser avec les images ». Comme historienne de l'art, je m'éloigne, tout comme Warburg, de la contemplation esthétisante des œuvres d'art et, comme lui, je m'empare du besoin d'image à l'œuvre dans les processus de pensée.

25. C'est-à-dire que ce soit «un répertoire de formules iconographiques figées, les symboles acquièrent une signification codée ou décodable, pouvant ainsi être facilement intégrés à un modèle iconographique traditionnel, comme cela se présente pour la majeure partie de la réception de Warburg ». S. Weigel, op. cit., p. 24.

26. Saccadé, en espagnol, évoque la "saccade électrique», mais aussi la discontinuité ( entrecortado » : ce qui est littéralement « entrecoupé »).

27. http://lemagazine.jeudepaume.org/2011/03/sabine-maria-schmidt-a-propos-demiddlemen-2001/

28. Cité par Sarah Ilher-Meyer, http://www.zerodeux.fr/specialweb/en-suspens-aernout-milkau-jeu-de-paume/

29. W. Benjamin, «Paralipomènes et variantes de l'œuvre d'art à l'époque de sa reproduction mécanisée », in Écrits français, Paris, Gallimard, 1991, pp. 225-226.

Selon Jean Maurice Monnoyer, « l'influence de Kracauer, qui prit la défense de Chaplin, n'est pas non plus discutable - les Paralipomènes qu'on lira en appendice indiquent même que la 
reconstruction mimétique du comportement mécanique est au centre de l'intuition chaplinesque ", Écrits français, op. cit., p.152.

30. Susan Buck-Morss: Voir le capital. Théorie critique et culture visuelle, Paris, Les Prairies Ordinaires, 2010. Voir, Walter Benjamin, "Sur quelques thèmes baudelairiens " in Euvres III, Paris, Gallimard, 2000, pp. 329-390.

31. « A lot of times I dance so fast that I become what's around me. It's like food for me, I like absorb that energy, absorb that information. It's like I eat it. It's the only now I get ».

32. Dominique Païni, Le Temps exposé. Le cinéma, de la salle au musée, Paris, Cahiers du Cinéma, coll. Essais, 2002, pp. 68-69.

33. «I take the extreme to instruct me as to what's most at stake with the mimetic faculty, this "degree zero" of similitude, an ineffable plasticity in the face of the world's forms and forms of life ", M. Taussig, Mimesis and Alterity. A Particular History of the Senses, New York et Londres, Routledge, 1993, p. 35.

34. « No proposition could be more fundamental to understanding the visceral bond connecting perceiver to perceived [...] in the operation of mimesis », Taussig, op. cit., p. 38.

35. P.-A. Michaud, Aby Warburg et l'image en mouvement, Paris, Macula, 1998.

36. Jean-Paul Colleyn, "Jean Rouch, presque un homme-siècle», in L'Homme, ed EHESS http:// lhomme.revues.org/document1545.html [page consultée le 02/02/2012].

37. Ibidem.

38. Cité dans Jean-Paul Colleyn: «Jean Rouch : cinéma et anthropologie » in Cahiers du Cinéma, 2009 , p. 31.

39. Nadine Wanono Sonchamp, « Jean Rouch ou la magie des images » in William Rothman (Ed.,) Jean Rouch a celebration of Life and Film, Transatlantique numéro 8, Janvier, 2007, Biblioteca della Ricerca, Schena Editore. Cité dans Paul Henley: The adventure of the real « The surrealist encounter" et "The coup de foudre on the rue Montparnasse ". wanono.org/.../ jean\%20rouch\%20la\%20magie...p...

40. J. Rouch, Les Maîtres fous, 1955, 36 min. Rouch a expliqué le double sens du titre : « ceux qui sont maîtres de leur folie, mais dont les maîtres sont fous ».

41. «La naissance des divinités Haouka est le symptôme d'un traumatisme provoqué par les pouvoirs militaires, politiques et techniques, dont elles sont des projections, mais aussi des formes de réappropriation mimétiques et magiques dans le rituel de possession ", G. Casas, «Petite histoire de la folie à l'âge postmoderne (note sur Les maîtres fous de Jean Rouch) » in MAG Philo, 2011. www.cndp.fr/magphilo/index.php?id=127

42. Les Maîtres fous, op. cit. : « [...] Le rite accompli, tout le monde se sépare, car il faut libérer les taxis loués pour la journée. Le lendemain, chacun a repris sa place au cœur des activités économiques de la ville ".

43. R. Sztutman, «Imagens perigosas : a possessao e a genesse do cinema do Jean Rouch» in Cadernos de campo, no 13, 2005. www.fflch.usp.br/da/cadcampo/ed_ant/revistas_completas/ 13.pdf. L'auteur cite la réflexion de Paul Stoller in Embodying Colonial Memories. Spirit Possession, Power and the Hauka in West Africa, New York et Londres, Routledge, 1995.

44. " L'image de substitution objective, l'excitation qui créé l'impression et en fait l'objet contre lequel on se défend. Par exemple, en voyant la mystérieuse locomotive comme un hippopotame, l'homme primitif lui confère un caractère contre lequel sa technique de combat lui permet de se défendre. Il pourrait l'abattre si elle se ruait vers lui ", Aby Warburg, Souvenirs d'un voyage en pays Pueblo (1923), trad. S. Muller, in Philippe-Alain Michaud, op. cit., p. 263.

45. J. Rouch, « Le vrai et le faux », p. 119.

46. Je cite aussi les mots de Warburg après son voyage chez les Hopi : "au lieu de le détruire, comme dans la civilisation moderne, en l'attirant à l'intérieur du sol au moyen d'un instrument inorganique. Ce qui distingue une telle attitude envers l'environnement de la nôtre, c'est que 
l'image mimétique doit chercher à obtenir cette relation de force, alors que nous visons la distance spirituelle et concrète. ", P. A. Michaud, op. cit., p. 259.

47. M. Rampley, The Remembrance of Things Past: On Aby M. Warburg and Walter Benjamin, Wiesbaden, Otto Harrassowitz, 2000.

48. "The gift of mimesis », dans les mots de K. Clark. Cité par G. Didi-Huberman, op. cit., p. 357. Je remercie Andrea Pinotti de m'avoir signalé, à l'occasion du colloque de Bordeaux, le caractère inapproprié de l'emploi du mot empathie (Einfühlung) que j'ôte désormais de mon texte. Je conserve l'expression de "champ sémantique » à propos du mimétisme, tout en reconnaissant qu'avec Les Maîtres fous, il ne s'agit pas d'une métamorphose mimétique propre à la danse culturelle aux masques.

49. M. Rampley, op. cit., p. 18: " Pour Benjamin, le sens des notions anthropologiques de la mimétique est bien connu. Le concept de mimesis est central dans son épistémologie ». Citation originelle : «The significance for Benjamin of such anthropological notions of the mimetic is well known. The concept of mimesis is central to his epistemology ".

50. Taussig, op. cit., p. 141.

51. Les figures prototypiques de la dynamique incorporative étaient l'enfant, le cannibale, l'acteur de cinéma et le clown. Voir, M. Bratu Hansen, Cinema and experience. Siegfried Kracauer, Walter Benjamin, and Theodor W. Adorno, University of California Press, 2011. Adorno avait dit : « Dans l'élément clownesque, l'art se souvient avec un sentiment de consolation de la préhistoire dans le monde primitif animal. Les singes (Menschenaffen) du zoo exécutent en commun ce qui ressemble à des actes clownesques. La connivence des enfants et des clowns est une connivence avec l'art, connivence que les adultes répriment, et tout autant une connivence avec les animaux. Le genre humain n'a pas réussi à se débarrasser si totalement de sa ressemblance avec les animaux qu'il ne puisse soudainement la reconnaître et en être submergé de bonheur ", T. W. Adorno, Théorie esthétique, Paris, Klincksieck, 1995 / GS 7 : 181-2.

52. W. Benjamin, « Sur le pouvoir d'imitation » in Euvres, t. II. Paris, Gallimard, 2000, p. 362.

53. Mik-who usually gives minimal instructions to his actors, preferring them to feel their way through their performance based on the relational dynamics of the groups involved-here asked a few performers to act out states of delirium in direct citation of scenes from an ethnographic film made in 1954 by Jean Rouch and titled Les Maîtres Fous (Mad Masters) (...) In Training Ground, Mik's actors manifest the same physical ailments that befall the possessed in Rouch's film. But here, the distinction between oppressor and oppressed becomes blurred to the point where we see police officers as well as refugees undergo spiritual entrancement. More importantly, though, the ritualistic aspects of Training Ground reveal the practice of exercise itself as representing a form of exorcism, albeit born out of fear and directed toward the future and not, as in Rouch's example, necessitated by the trauma of a time past. Claudia Schmuckli, Rituals of Engagement, http://www.worldclassboxing.org/exhibit_amik.php

54. M. Taussig, dans "Reflexiones", in Miradas cruzadas. Cine y antropología, Madrid, La Casa Encendida, 2007, p. 141, parle d'un " excès mimétique » ou d'une " mimesis auto-réfléchie »: « Une mimesis cosnciente d'elle-même grâce à la fusion de la machine mimétique avec le corps dansant mimétique. L'image se transforme en chair animée et l'imitation se réalise par contact. Cela nous rapproche davantage de ce que Callois (...) décrivait comme similaire ». Citation originelle : «Una mímesis consciente de sí misma gracias a la fusión de la máquina mimética con el cuerpo danzante mimético. La imagen se convierte en carne en movimiento y la imitación se realiza por contacto. Esto nos acerca aún más a lo que Caillois [...] describía como similar ».

55. Taussig, ibidem.

56. J. Colleyn (ed.) (2009), Jean Rouch. Cinéma et anthropologie, Paris, Cahiers du cinéma-INA, 2009.

57. Colleyn, op. cit.

58. A. Artaud, «Sorcellerie et cinéma », 1927, extrait du livre d'Antonin Artaud, Oeuvres complètes

III, Gallimard, 1978. http://www.derives.tv/Sorcellerie-et-Cinema 
59. J. Rouch, cité par Taussig, op. cit., p. 141.

60. J. Rouch « Le vrai et le faux », op. cit.

61. Taussig, op., cit., p. 140.

62. Taussig, op. cit., p. 143.

63. H. Bhabha, Les Lieux de la culture. Une théorie postcoloniale, Paris, Payot, 2007.

64. « Le rite accompli, tout le monde se sépare, car il faut libérer les taxis loués pour la journée. Le lendemain, chacun a repris sa place au cœur des activités économiques de la ville».

65. Je remercie Vincent Meessen de m'avoir signalé que Rouch lui-même dira « plus tard qu'il n'aime pas la fin de son commentaire ».

66. Voir Lim Kien Ket, " Of mimicry and White man. A psycoanalysis of Jean Rouch's Les Maîtres fous ", Cultural Critique 51, printemps 2002. Pour la confrontation entre Rouch et Sembéne réalisée par A. Cervoni, dans France Nouvelle $\mathrm{n}^{\circ} 1033,4$ août 1965, voir http://www.jstor.org/discover/10.2307/1354636?

uid $=3737952 \&$ uid $=2129 \&$ uid $=2 \&$ uid $=70 \&$ uid $=4 \&$ sid $=56135794363$

67. J. J. de Carvalho, "La mirada etnográfica y la voz subalterna ", Revista colombiana de antropología, vol. 38, 2002, p. 311. redalyc.uaemex.mx/redalyc/pdf/1050/105015289012.pdf

68. H. Bhabha, op. cit., p. 83.

69. «The image-world is the surface of globalization. It is our shared world. Impoverished, dim, superficial, this image surface is all we have of shared experience. Otherwise we do not share a world », S. Buck-Morss, «Visual Studies and global imagination », Papers of Surrealism Issue 2, summer 2004, p.25. www.surrealismcentre.ac.uk/papersofsurrealism/journal2/acrobat_files/ buck_morss_article.pdf

70. G. Didi-Huberman, op. cit., p. 48 et pp. 103-104.

\section{INDEX}

Mots-clés : Altération, absorption, mimétisme, environnement, technologie, choc, schizophrénie, civilisation occidentale

\section{AUTEUR}

\section{AURORA FERNÁNDEZ-POLANCO}

Professeure de Théorie et Histoire de l'art contemporain à l'Université Complutense de Madrid. Parmi ses publications récentes : «Voir Basilio Martin Patino avec Georges Didi-Huberman », in T. Davila et P. Sauvanet (dir.), Devant les images. Penser l'art et l'histoire avec Georges DidiHuberman, Dijon, Les Presses du réel, 2011 ; « Ver a distancia », in S. Aznar et P. Martinez (dir.), Lecturas para un espectador inquieto, Madrid, CA2M, 2012. Elle est éditrice de la revue Re-visiones) 